\title{
Beneficial effects of small-sided games as a conclusive part of warm- up routines in handball
}

Antonio Dello Iacono ${ }^{1,2 *}$, Andrew D. Vigotsky ${ }^{3}$, Lior Laver ${ }^{4,5}$, Israel Halperin ${ }^{6}$

${ }^{1}$ Department of Life Sciences, The Academic College at Wingate, Wingate Institute, Israel

${ }^{2}$ Sport Science Department, Maccabi Tel Aviv FC, Tel Aviv, Israel

${ }^{3}$ Department of Biomedical Engineering, Northwestern University, Evanston, IL, USA

${ }^{4}$ Department of Orthopedic Surgery, Faculty of Medicine, Tel Aviv University, Tel Aviv, Israel.

${ }^{5}$ Department of Trauma and Orthopaedics, University Hospitals Coventry and Warwickshire, Coventry, UK.

$12{ }^{6}$ School of Human Kinetics and Recreation, Memorial University of Newfoundland, St. John's,

13 Canada

14

15 *Correspondence:

16 Dr. Antonio Dello Iacono

17 badinbi@gmail.com 


\begin{abstract}
The aim of this study was to compare the effects of small sided games (SSG) and traditional warm-up strategies on the mechanical, physiological, and perceptional responses of handball players. Using a randomized and counterbalanced design, 12 elite male handball players completed a general 8 min warm-up which was concluded with an 8 min section of either specific handball shooting drills or $3 \times 2$ min of SSG with a passive recovery of 1 min between bouts. Countermovement jumps and plyometric press-ups were assessed before and immediately after the warm-up regimens using a force plate. Heart rate (HR) was assessed during the warm up regimens, and rating of perceived effort (RPE) was assessed after the regimens. Meaningful differences favoring SSG were observed in most of the kinetic variables in the countermovement jumps and plyometric press-ups ( $\mid$ Hedges' $g \mid=0.26-1.42$ ). Conversely, no meaningful differences were found between warm up regimens in RPE or HR responses $(z$-scores $=0.45$ and 1.88 , respectively). These results indicate that concluding warm-ups with SSGs offer greater benefits compared to a more traditional warm-up routine, despite similar HR and RPE responses even when matched for duration among elite level handball players.
\end{abstract}

Keywords: jumps; potentiation; press-up; small sided games; team sport; video analysis 


\section{Introduction}

Warm-up routines are widely implemented prior to athletic competition as means to optimize performance. A recent meta-analysis reported that $79 \%$ of the analyzed studies observed performance enhancing effects following various warm-up protocols (Fradkin et al., 2010). The proposed mechanisms accounting for the benefits of warm-ups are physiological, mechanical, and psychological in nature (McGowan et al., 2015). While the practical benefits and underpinning mechanisms of warm-ups are generally agreed upon, the particular implementation strategies are still debated. In team sports, warm-ups may last up to $40 \mathrm{~min}$ (Romaratezabala et al., 2018) and are traditionally structured by including sequences of moderate- to high-intensity and generic to specific activities (McGowan et al., 2015). However, evidence suggests that shorter-duration (10-15 min) activities, completed at $40-70 \%$ of maximal rate of oxygen consumption, may be sufficient to improve short, intermediate, and long-term performance (Bishop, 2003). Reviewing the warm-up literature, McGowan et al. (2015) concluded that prematch warm-up strategies for team sports that aim to optimize subsequent performances should employ sport-specific activities, while keeping the total effective duration of less than 16 min. As such, shortening the duration of the warm-up and making it more sport-specific may further improve its benefits, both of which can be achieved by implementing small-sided games (SSG) as a warm-up strategy.

SSG can be defined as team-sport games, performed in small playing areas with a reduced number of players, variable rules, and technical constraints. These games are designed to simulate both the technical/tactical and physical/physiological demands of a particular discipline (Halouani et al., 2014). SSG are beneficial insofar as they can replicate the movement patterns, physiological demands, and technical requirements of competitive matches (Buchheit et al., 2009), and by targeting all related warm-up mechanisms in a sport-specific manner. To date, only two studies compared the effects of SSG as a warm-up strategy to a traditional warm-up routine on subsequent athletic task performances, but with mixed results. Gabbett et al. (2008) investigated the effects of open skill activities as a warm-up strategy for basketball players, and observed no differences in comparison to a traditional warm-up, as measured by reactive agility, vertical jump, and sprint performance. The open-skill activities included a variety of technical high-intensity actions performed individually which progressed into 4 vs. 4 SSG. Conversely, Zois et al. (2011) found that using 3 vs. 3 SSG improved countermovement jump (CMJ), repeated sprint, reactive agility, and $40 \mathrm{~m}$ sprint performance among soccer players compared to a traditional warm-up. These conflicting effects could have stemmed from the different durations of the warm-up periods in the two studies. Whereas the overall duration of the warm-up in Gabbett's study was 22 min, in which the SSG part lasted 15 min, the warm-up duration in Zois`s study lasted approximately $12 \mathrm{~min}$ in total, with only 6 min dedicated for the SSG. The longduration of high-intensity warm-ups in Gabbett's study may have compromised the enhancement of subsequent performance (McGowan et al., 2015).

Considering the potential SSG have as a warm-up strategy and the dearth of studies on this topic, with existing evidence reporting mixed effects, there is a need to further explore and compare the inclusion of SSG in warm up regimens to traditional warm-ups. Accordingly, the goal of this study was to compare the acute effects of SSG-based warm-up and a traditional warm-up on upper and lower body athletic performances and the associated mechanical, physiological, and perceptional outcomes in elite handball players. 


\section{Material and Methods}

\section{Study Design}

A randomized, crossover study design was implemented, in which mechanical, physiological, and perceptual responses (Foster et al., 2001) were monitored before and immediately after either traditional or SSG-based warm-up protocols. Athletes randomly completed two experimental trials five days apart. The first half of the warm-up protocol was similar in both conditions and was composed of standardized handball pre-match warm-ups lasting roughly 8 min. Subsequently, athletes completed a baseline countermovement (CMJ) and plyometric pressup (PP) assessment, followed by either handball-specific shooting drills or 3-a-side handball SSG for $8 \mathrm{~min}$. The same CMJ and PP tests were then repeated 7 min following the warm-up regimens (Bishop, 2003), during which the athletes stood or walked. The order in which the tests were completed was counterbalanced for each participant and the order of conditions was determined by block randomization (Schulz and Grimes, 2002) using an online randomization tool. Two weeks prior to the study, participants performed two familiarization sessions 5 days apart. On these occasions, the exact experimental sequences, including baseline assessment, either traditional or handball specific SSG-based warm-up protocols, and post warm-up assessment were followed.

\section{Subjects}

A convenience sample of twelve elite male handball players belonging to a U-21 national handball team (age $19.3 \pm 0.4$ years; height $186.5 \pm 8.4 \mathrm{~cm}$; weight $86.8 \pm 8.4 \mathrm{~kg}$ ) participated in the study. The participants had $8.2 \pm 1.5$ years of training experience, and practiced 8 times per week for a total of $11.5 \pm 1.1$ hours of weekly handball activities. Written informed consent was obtained from each participant after receiving an explanation of the purpose, benefits, and potential risks of the study. This study was carried out in accordance with the recommendations of the Declaration of Helsinki after being approved by the ethical committee for human research of the Academic College at Wingate, Israel.

\section{Procedures}

Five days prior to the first experimental trial, measurements of height and body mass (SECA model 284, Germany) were taken. On the same day, the Yo-Yo intermittent recovery test level 1 (YYIRTL1) (Souhail et al., 2010) was performed to determine the maximum heart rate $\left(\mathrm{HR}_{\max }\right)$ values, which were used for the calculation of exercise intensity during the two experimental warm-up sessions. All testing sessions were performed on a regular indoor court and completed at the same time of the day (11:00 a.m. - 13:00 p.m.) and in similar ambient conditions of temperature $\left(20.5 \pm 0.5^{\circ} \mathrm{C}\right)$ and relative humidity $(60 \pm 4.5 \%)$. In order to prevent unnecessary fatigue, players and coaches were instructed to avoid intense training $24 \mathrm{~h}$ prior to each testing day. Additionally, participants were also asked to avoid eating $2 \mathrm{~h}$ before each testing sessions.

\section{Lower body mechanical assessment}

Lower body mechanical capabilities were assessed by a CMJ test according to the protocol previously described by Dello Iacono et al. (2017). Each subject performed a bilateral CMJ on a force plate (Kistler Biomechanics, Winterthur, Switzerland) starting from a stationary position 
with their hands on the waist and knees fully extended, squatting down to about $\sim 90^{\circ}$ of knee flexion before starting an explosive, upward motion, with the intent to jump as high as possible. Knowledge of jump performance was provided to the athletes after each jump, and verbal encouragement was provided prior to each trial by two research assistants, who were blinded to which group each subject was assigned. Subjects carried out three maximal CMJ with 30-45 s rest between each jump to avoid fatigue.

The vertical ground reaction force signal $\left(F_{z}\right)$ was acquired from the Kistler amplifier to a 12-bit A/D converter at $1000 \mathrm{~Hz}$ sampling rate and stored on a PC (Dell Inspiron 9100, Dell, United Kingdom). Data were filtered through a fourth-order, low-pass Butterworth digital filter, with a cutoff frequency of $100 \mathrm{~Hz}$. The $\mathrm{F}_{\mathrm{z}}$ signal was later analyzed according to the methods described by Caserotti et al., (2001) and the following parameters were calculated: peak eccentric force $\left(\mathrm{F}_{\text {peak-ecc }}\right)$, peak concentric force $\left(\mathrm{F}_{\text {peak-con }}\right)$, peak concentric power $\left(\mathrm{P}_{\text {peak-con }}\right)$, jump height $(\mathrm{JH})$, and displacement of the body center of mass during the eccentric $\left(\mathrm{S}_{\mathrm{ecc}}\right)$ phase. Specifically, jump power was calculated as the instantaneous product between the $F_{z}$ and the vertical velocity $(v)$ of the center of mass; force and velocity in other planes were assumed to be negligible. The latter was obtained by time integration of the instantaneous acceleration:

$$
v(t)=\int_{0}^{t} a_{n e t}(t) d t=\int_{0}^{t}\left[F_{z}(t) / m-g\right] d t
$$

where $a_{n e t}$ is the vertical acceleration, $F_{z}$ is the vertical force measured by the platform, $m$ is the body mass of the subject, and $g$ is the acceleration due to the gravity $\left(9.81 \mathrm{~m} / \mathrm{s}^{2}\right)$. Center of mass position was plotted throughout the whole movement by time integration of the velocity signal. Finally, jump height was measured from the value of vertical velocity at take-off point ( $\left.\mathrm{v}_{\text {takeoff }}\right)$ :

$$
\text { jump height }=\frac{v_{\text {takeoff }}^{2}}{2 g}
$$

\section{Upper body mechanical assessment}

Upper body mechanical capabilities were assessed by PP according to the protocol of Johnston et al. (2013). Players were asked to start in a press-up position with their hands on the force platform in a self-selected position and arms extended. On the experimenter's signal, the players were lowered their body by flexing their elbows to a self-selected depth before extending the elbows as quickly as possible so that their hands left the platform. Subjects performed two selfchosen trials without data being recorded before completing three PP with 30-45 s of rest between them. The same procedural methods described above for the lower body mechanical responses assessment were applied (Dello Iacono et al., 2017). All PP trials were analyzed for the eccentric and the concentric phases and the kinetic parameters selected for further analysis were: peak eccentric force $\left(\mathrm{F}_{\text {peak-ecc }}\right)$, peak concentric force $\left(\mathrm{F}_{\text {peak-con }}\right)$, and peak concentric power $\left(\mathrm{P}_{\text {peak-con }}\right)$.

\section{Warm-up protocols}

Proceeding each experimental session, participants underwent a 8 min standardized warm-up: 3 min of locomotor activities including jogging, running, and multidirectional changes of direction (COD); 3 min of dynamic stretching exercises for the upper and lower extremities; and 2 min of 
jumping drills and press-up exercises, accelerations, and short-sprint drills over a distance of 20 $\mathrm{m}$. Shortly after an active recovery ( 2 min), participants completed baseline assessment including the CMJ and PP. Immediately after, the players completed either handball-specific shooting warm-up drills or SSG for additional $8 \mathrm{~min}$. The handball-specific shooting warm-up was composed of:

- Short $(5 \mathrm{~m})$ to long distance $(20 \mathrm{~m})$ passes (both with one and two arms performed as overhead or side ones), completed in couples;

- Five free shots performed from the $9 \mathrm{~m}$ line with increasing intensity up to the maximal shooting effort.

- Five jump shots performed from the players` own position and preceded by a run-in of about $5 \mathrm{~m}$ (Wagner and Muller, 2008).

Finally, about $7 \mathrm{~min}$ following the shooting warm-up, the assessment procedures were repeated.

The SSG warm-up was composed of: regular small-sided handball matches and were organized in 3-a-side teams including goalkeepers (Iacono et al., 2015; Dello Iacono et al., 2016; Dello Iacono et al., 2017; Dello Iacono et al., 2018). The SSG warm-up consisted of $3 \times 2$ min bouts with a passive recovery of $1 \mathrm{~min}$ between bouts played on a regular handball court $(40 \mathrm{~m} \times 20$ $\mathrm{m})$. Some playing rules were created in order to avoid game breaks ensuring continuity and high exercise intensity. For instance, standing and dribbling were not allowed, defense stops due to regular fouls were sanctioned with ball turnover, the maximal time to complete an attack before losing ball possession was preset at $20 \mathrm{~s}$. Additionally, several balls were placed around the sided games area for immediate availability in order to avoid game pause. Finally, in case of a penalty, the latter was considered as a regular goal and no free shot was given. To avoid any bias due to the players' anthropometric, physiological positional role characteristics, the four teams were equally lined up, including one wing player, one line player/pivot, and one back player (Chaouachi et al., 2009).

\section{Heart rate responses}

HR responses were monitored during both the traditional and the specific SSG-based warm-up to provide the mean heart rate percentage $\left(\% \mathrm{HR}_{\text {mean }}\right)$ because it is better indicative of what occurs over the entire warm-up as compared to $\mathrm{HR}_{\text {max }}$. $\mathrm{HR}$ responses were recorded via a telemetry system (Hosand Technologies Srl, Verbania, Italy) at $5 \mathrm{~s}$ intervals throughout each SSG and then filtered using a software-embedded proprietary algorithm (Hosand MC Software ${ }^{\mathrm{TM}}$, Verbania, Italy). The $\% \mathrm{HR}_{\text {mean }}$ and the $\mathrm{HR}_{\max }$ were considered the corresponding values measured at the end of the YYIRTL1 test.

\section{Rating of perceived effort (RPE)}

Players indicated their rating of perceived exertion (RPE) using the category rating 10 (CR-10) scale modified by Foster et al. (2001) at the end of the experimental session, using a standardized questionnaire.

\section{Statistics}

To assess the effects of the warm-ups, all data were imported to $\mathrm{R}$ (version 3.4.3) for analyses (Team, 2013). For data that consisted of pre- and post-testing $\left(\mathrm{JH} ; \mathrm{S}_{\mathrm{ecc}}\right.$ during the CMJ; $\mathrm{F}_{\text {peak-con }}$ and $\mathrm{F}_{\text {peak-ecc }}$ during the $\mathrm{CMJ}$ and $\mathrm{PP}$; and $\mathrm{P}_{\text {peak-con }}$ during the $\mathrm{CMJ}$ and $\left.\mathrm{PP}\right)$, linear mixed-effects 
models were used (Bates et al., 2015). Using the effect of condition (i.e., the effect of SSG relative to control), Hedges' $g$ effect sizes were calculated to estimate a conservative standardized mean difference (Hedges, 1981; Lakens, 2013). Finally, 95\% percent confidence intervals (CI) were calculated for the condition effects.

Rating of perceived exertion and HR data were analyzed using permutation tests, which is a nonparametric testing paradigm that compares the observed effects to a simulated null distribution. By comparing the observed effects to the null distribution, a $z$-score and $p$-value could be calculated. Finally, 95\% CIs were calculated around the observed effect.

To avoid dichotomous interpretations of the results, no a priori $\alpha$-level was set. Rather than interpreting effects from a single test, or set of tests, the results were interpreted on a continuum using all statistical outcomes, in combination with theory and practical considerations (McShane, 2017). Due to the unconventional nature of these procedures in our field, we have supplied Supplementary Materials and analysis code, which extensively detail these statistics and allow for the reader to reproduce them.

\section{Results}

Linear mixed-effects models revealed marked improvements in $\mathrm{JH}, \mathrm{S}_{\text {ecc }}$ during the CMJ, $\mathrm{F}_{\text {peak-ecc }}$

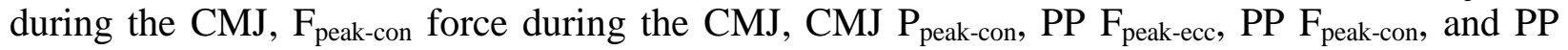
$\mathrm{P}_{\text {peak-con }}$ relative to the control warm-up (Table 1 and Figures 1 and 2). Permutation tests did not reveal any remarkable differences between SSG and warm-up conditions in RPE or mean HR (Table 1).

Table 1. Performance and exertional outcomes between SSG and control warm-ups

\begin{tabular}{|c|c|c|c|c|c|}
\hline Outcome & Effect & $95 \% \mathrm{CI}$ & Hedges' $g$ & $z$-score & $p$-value \\
\hline Jump height (cm) & 2.67 & $1.99-3.33$ & 1.23 & & $<0.001$ \\
\hline Eccentric displacement $(\mathrm{cm})$ & -2.19 & $-3.45--0.74$ & -0.26 & & 0.010 \\
\hline CMJ F $F_{\text {peak-ecc }}(\mathrm{N} / \mathrm{kg})$ & 3.05 & $2.12-3.85$ & 1.30 & & $<0.001$ \\
\hline CMJ F $F_{\text {peak-con }}(\mathrm{N} / \mathrm{kg})$ & 2.45 & $2.01-2.89$ & 1.42 & & $<0.001$ \\
\hline CMJ $P_{\text {peak-con }}(\mathrm{W} / \mathrm{kg})$ & 3.02 & $2.29-3.71$ & 1.42 & & $<0.001$ \\
\hline PP F $F_{\text {peak-ecc }}(\mathrm{N} / \mathrm{kg})$ & 1.52 & $1.20-1.85$ & 1.21 & & $<0.001$ \\
\hline PP F $F_{\text {peak-con }}(\mathrm{N} / \mathrm{kg})$ & 1.73 & $1.18-2.28$ & 1.20 & & $<0.001$ \\
\hline PP $P_{\text {peak-con }}(\mathrm{W} / \mathrm{kg})$ & 2.06 & $1.28-2.86$ & 1.06 & & $<0.001$ \\
\hline RPE (AU) & 0.08 & $-0.25-0.42$ & & 0.45 & 0.997 \\
\hline Mean HR (\%) & 1.42 & $0.08-2.58$ & & 1.88 & 0.077 \\
\hline
\end{tabular}

Effect represents the estimate of the effect of performing a SSG warm-up relative to a control warm-up (i.e., effect $=\mathrm{SSG}-\mathrm{Control}$ ). $\mathrm{CI}=$ confidence interval; $\mathrm{CMJ}=$ countermovement jump; $\mathrm{PP}=$ plyometric press-up; $\mathrm{RPE}=$ rating of perceived exertion; $\mathrm{HR}=$ heart rate. 

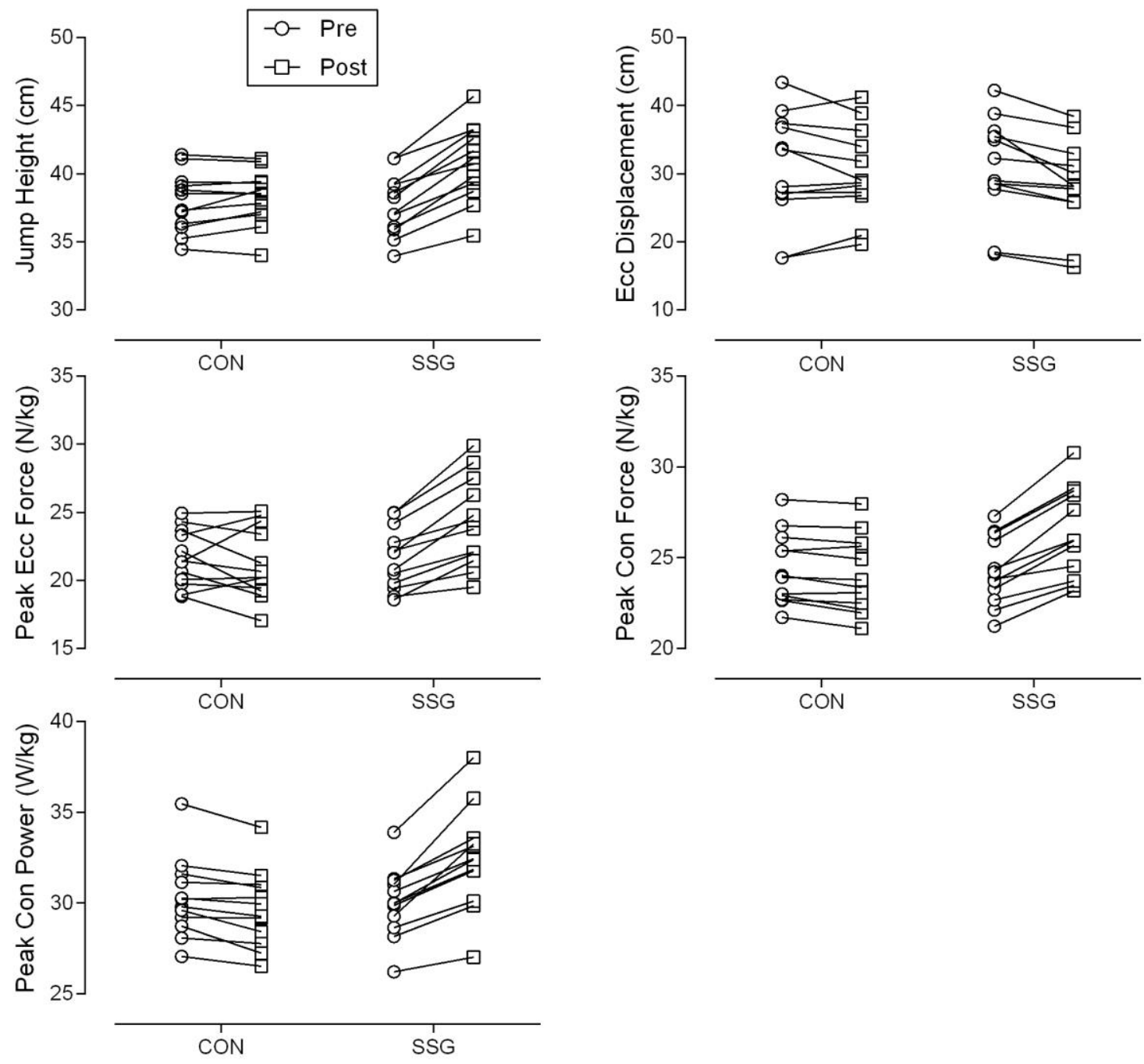

Figure 1: Plot of the CMJ mechanical responses changes following the traditional $(\mathrm{CON})$ and SSG-based (SSG) warm-up protocols 

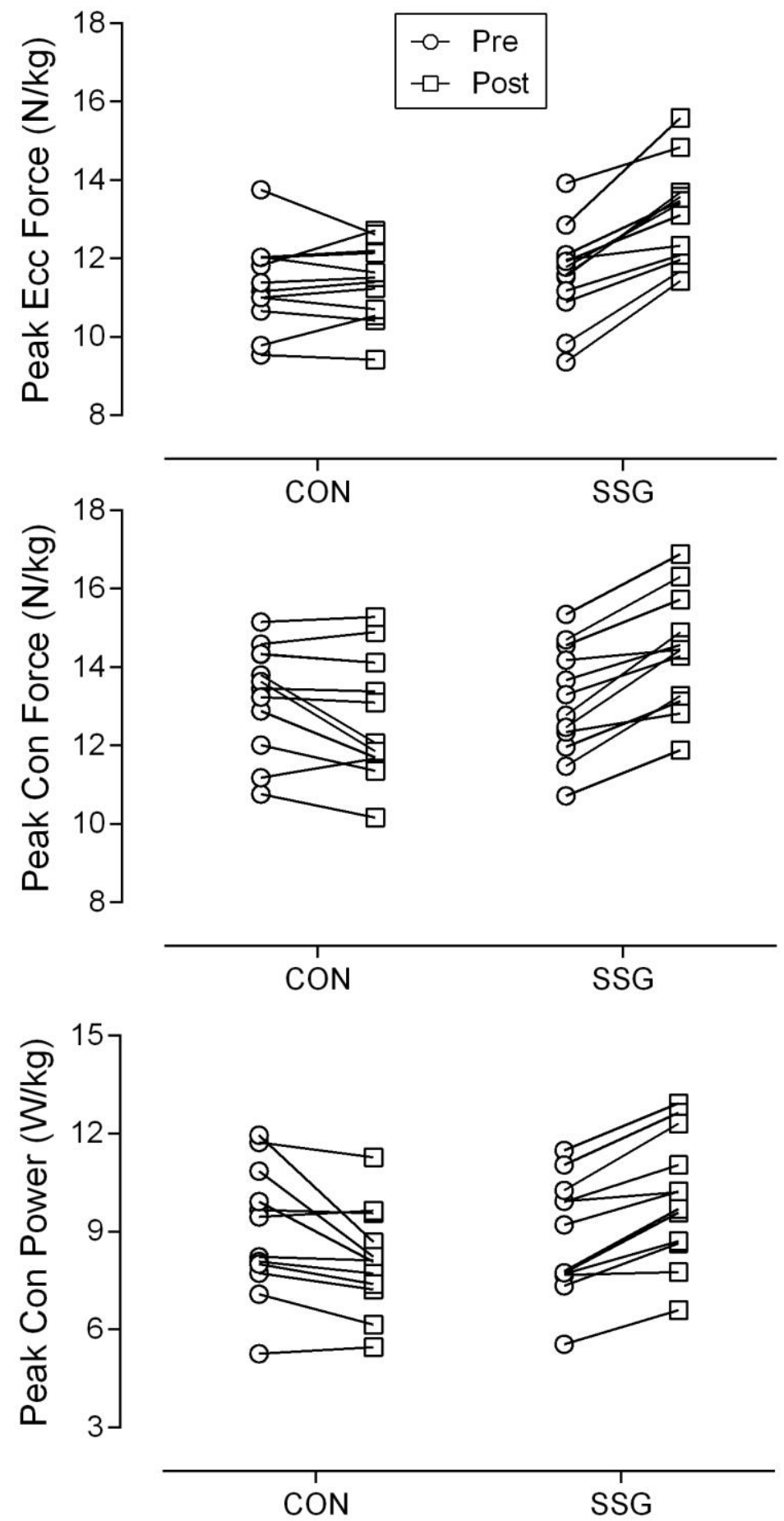

246 Figure 2. Plot of the PP mechanical responses changes following the traditional (CON) and 247 SSG-based (SSG) warm-up protocols 


\section{Discussion}

The SSG warm-up was found to result in superior outcomes for both upper and lower body even when considering that duration, intensity, and perceptional responses of the two routines were similar. To our knowledge, this is the first study to have examined mechanical responses and physiological mechanisms underlying changes in the assessed athletic performances of SSG warm-up.

\section{Lower body}

The SSG-based warm-up enhanced CMJ height by $7.1 \%$ compared to the traditional warm-up routine and was associated with greater improvements in all the kinetic measures. Specifically,

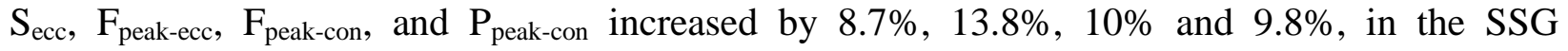
compared to the traditional warm-up routine, respectively (Figure 1). These results are in line with some, but not all investigations. Whereas the results of the current study are in line with those reported by Zois et al., (2011), which observed a similar 6\% improvement in CMJ following the SSG warm-up, Gabbett et al., (2008) did not observe an advantage of open skillbased warm-up including SSG over a traditional warm-up routine on various performance outcome measures. This discrepancy could stem from dissimilar durations of the warm-up in these studies. Whereas the duration of the warm-up in present study and the study by Zois et al. (2011) lasted 12-16 min, a duration that is in line with published recommendations, Gabbett and colleagues (2008) implemented a 22 min warm-up, which may have hindered subsequent performance.

The enhancement of the CMJ performance following the SSG-based warm-up may in part be explained by a post activation potentiation (PAP) effect (Suchomel et al., 2016). The frequent "one-on-one" SSG-related playing situations involve dynamic actions such as accelerations, decelerations, change of directions, and jumps. These actions are ballistic in nature and previous research has indicated that ballistic movements produce greater muscle and power outputs than non-ballistic ones (Newton et al., 1997; Lake et al., 2012). As a consequence, they may have induced activation of high-threshold motor units, which have been proposed to underline the PAP (Tillin and Bishop, 2009). Our results are also consistent with the observations of Wilson et al. (2013), showing that multiple sets of the potentiating activities, and recovery intervals of 5-7 min between the conditioning activity and the subsequent performance induce large PAP effects. Therefore, we suggest that the short duration of the SSG-based warmup protocol $(8 \mathrm{~min})$ organized in a multiple-set format $(3 \times 2 \mathrm{~min})$ and the time scheduling relative to the functional performance $(7 \mathrm{~min})$ have likely influenced the magnitude of the effects on muscle mechanical enhancements. The lack of differences in the HR and RPE responses between the two warm-up regimens strengthens the possibility that increases in neural activation associated to the PAP effects accounted for the superior SSG warm-up performance.

Interestingly, the changes in the mechanical responses following the SSG-based warm-up suggest small modifications in jumping strategy. During the eccentric phase of the CMJ, athletes reduced the downward displacement while developing greater force and power outputs during both the downward and the subsequent upward actions (Figure 1). The possible underlying mechanisms leading to these effects may be related to modifications in the characteristics of the joints involved and muscles recruited in performing the CMJ task. Following the SSG-based warm-up, the lower limbs kinematics may have changed, thus resulting in increased joint velocities and improved joint coupling (Kawakami et al., 2002; Suchomel et al., 2016). Such modifications of the jump strategy and the required motor control are presumably responsible of 
the reduced downward displacement during the CMJ after the SSG-based warm-up. In addition, possibly parallel changes of the muscle-related characteristic, like increases of the motor unit recruitment and discharge rates, changes of the muscles` architecture (Mahlfeld et al., 2004; Fukashiro et al., 2006) and muscle stiffness (Aagaard et al., 2002; Tillin and Bishop, 2009), may have contributed to the increase in strength and power outputs. However, because no direct neurophysiological measurements were collected in this study, the two assumptions accounting for the improved mechanical outputs are speculative and should be explored by future research in order to be better understood. Additionally, the size of the effect was small and as such, it is not clear if the identified differences in jump strategy represent a meaningful, replicable effect.

\section{Upper body}

The SSG-based warm-up led to greater improvements in all the mechanical measures in the PP in comparison to the traditional warm-up protocol. $\mathrm{F}_{\text {peak-ecc }}, \mathrm{F}_{\text {peak-con, and }} \mathrm{P}_{\text {peak-con }}$ increased by $12.9 \%, 13.3 \%$, and $22.8 \%$, respectively in the SSG warm-up relative to the traditional warm-up (Figures 2). To our knowledge, no other study examined and compared the effects of SSG and traditional warm-up on explosive upper body exercises, making direct and contrasting comparisons impossible. It can be assumed that common handball specific physical contact actions such as hitting, blocking, pushing, and holding occurring between players in the SSG warm-up in addition to shooting and passing, could have generated a PAP effect on the PP task. This is especially the case when considering that such actions are completed against heavy resistance, as represented by the opponent's mass, a condition that was absent from the traditional warm-up routine. Accordingly, the high similarity between the upper body actions demanded during handball SSG and the assessed PP task together with a proper mechanical overloading may have produced PAP.

There is clear evidence of the chronic effects of contact SSG on upper body neuromuscular performance in handball players. Iacono et al. (2015) observed that SSG training created a cumulative training stimulus that produced improvements in upper body strength, as measured by $1 \mathrm{RM}$ bench press test over a period of 8 weeks. However, the same authors (Dello Iacono et al., 2017) recently demonstrated that a SSG lasting $15 \mathrm{~min}$ led to acute impairments in upper body mechanical performances during the following PP task. The performance decrements reported by Dello Iacono et al. (2017) are likely due to the longer duration of the SGG in comparison to the warm-up protocol used in the current study (15 vs. 6 min). These results indicate that the presence of an adequate amount of actions associated to physical contacts during SSG determines ergogenic effects on upper body musculature.

Altogether, these findings may have immediate implications for the implementation of SSG formats into a warm-up routine. Besides the observed positive effects on the subsequent CMJ and PP performances, the opportunity to easily schedule this warm-up protocol, using sport-specific drills, in the imminence of the competitive activity, may likely improve both players' compliance and coaches' acceptance. We therefore recommend the SSG-based warm-up routine implemented in the current study to be used prior to handball competitions be performed, since mechanical adaptations may be achieved and beneficial effects on the consequent functional performances may be obtained.

There are a number of limitations in this study. First, due to logistical constraints, only two outcome measures were collected, both of which are indirectly related to handball performance. Future studies should also measure more sport-specific tests, such as agility tests and ball throwing velocities. Second, the 7 min delay between the end of the warm-up routines 
and the initiation of the testing procedures may have washed out some of the warm-up effects. Despite this limitation, this testing procedure has a higher degree of ecological validity as it mimics the common behaviors of team sport associated with 5-10 minutes delay between the end of the warm-up and beginning of a game (McGowan et al., 2015). Finally, we did not conduct a power analysis to determine the small size. This is because the population from which welltrained handball players can be drawn, belonging to the same team and with a common training background is limited. To overcome this problem, we conducted a within-subject design, and attempted to reduce learning curves by including familiarization sessions.

\section{Conclusions}

In conclusion, the results of this study point to the effectiveness of SSG as a part of a warm-up strategy. Compared to a more traditional handball warm-up routine, SSG led to superior performance and mechanical responses of handball athletes, even when the total warmup duration was controlled for, and no differences in HR and RPE responses were identified between conditions. We speculate that the superior effects of the SSG warm-up are related to PAP effects; however, the underlying mechanisms remain to be directly investigated. Coaches should consider implementing SSG not only as a training strategy, but also as part of a warm-up strategy considering its beneficial effects on relevant athletic performance.

\section{Author Contribution Statements}

ADI, LL made substantial contributions to the conception and design of the study; ADI, LL supervised the experimental sessions and contributed to data collection; AV, IH carried out data analysis and interpretation of the results together with ADI and LL; ADI Wrote the first draft of the manuscript and all authors were involved in revising it critically for important intellectual content; AV, LL and IH: Gave final approval of the version to be published and agreed to be accountable for all aspects of the work.

\section{Conflict of Interest Statement}

The authors declare that the research was conducted in the absence of any commercial or financial relationships that could be construed as a potential conflict of interest

\section{Acknowledgements}

The authors wish to thank Yochai Wiener for assisting with the data collection during the experiments

\section{Funding disclosure \\ No funding has been obtained for this study}

\section{Data Availability Statements}

The raw data supporting the conclusions of this manuscript will be made available by the authors, without undue reservation, to any qualified researcher. 


\section{Specific warm-up in team handball}

\section{References}

Aagaard, P., Simonsen, E.B., Andersen, J.L., Magnusson, P., and Dyhre-Poulsen, P. (2002). Increased rate of force development and neural drive of human skeletal muscle following resistance training. J Appl Physiol (1985) 93(4), 1318-1326. doi: 10.1152/japplphysiol.00283.2002.

Bates, D., Mächler, M., Bolker, B., and Walker, S. (2015). Fitting Linear Mixed-Effects Models Using lme4. 2015 67(1), 48. doi: 10.18637/jss.v067.i01.

Bishop, D. (2003). Warm up II: performance changes following active warm up and how to structure the warm up. Sports Med 33(7), 483-498.

Buchheit, M., Laursen, P.B., Kuhnle, J., Ruch, D., Renaud, C., and Ahmaidi, S. (2009). Gamebased training in young elite handball players. Int J Sports Med 30(4), 251-258. doi: 10.1055/s-0028-1105943.

Caserotti, P., Aagaard, P., Simonsen, E.B., and Puggaard, L. (2001). Contraction-specific differences in maximal muscle power during stretch-shortening cycle movements in elderly males and females. Eur J Appl Physiol 84(3), 206-212. doi: 10.1007/s004210170006.

Chaouachi, A., Brughelli, M., Levin, G., Boudhina, N.B., Cronin, J., and Chamari, K. (2009). Anthropometric, physiological and performance characteristics of elite team-handball players. J Sports Sci 27(2), 151-157. doi: 10.1080/02640410802448731.

Dello Iacono, A., Ardigo, L.P., Meckel, Y., and Padulo, J. (2016). Effect of Small-Sided Games and Repeated Shuffle Sprint Training on Physical Performance in Elite Handball Players. J Strength Cond Res 30(3), 830-840. doi: 10.1519/jsc.0000000000001139.

Dello Iacono, A., Eliakim, A., Padulo, J., Laver, L., Ben-Zaken, S., and Meckel, Y. (2017). Neuromuscular and inflammatory responses to handball small-sided games: the effects of physical contact. Scand J Med Sci Sports 27(10), 1122-1129. doi: 10.1111/sms.12755.

Dello Iacono, A., Martone, D., Zagatto, A.M., Meckel, Y., Sindiani, M., Milic, M., et al. (2018). Effect of contact and no-contact small-sided games on elite handball players. J Sports Sci 36(1), 14-22. doi: 10.1080/02640414.2016.1276296.

Foster, C., Florhaug, J.A., Franklin, J., Gottschall, L., Hrovatin, L.A., Parker, S., et al. (2001). A new approach to monitoring exercise training. J Strength Cond Res 15(1), 109-115.

Fradkin, A.J., Zazryn, T.R., and Smoliga, J.M. (2010). Effects of warming-up on physical performance: a systematic review with meta-analysis. J Strength Cond Res 24(1), 140148. doi: 10.1519/JSC.0b013e3181c643a0.

Fukashiro, S., Hay, D.C., and Nagano, A. (2006). Biomechanical behavior of muscle-tendon complex during dynamic human movements. J Appl Biomech 22(2), 131-147.

Gabbett, T.J., Sheppard, J.M., Pritchard-Peschek, K.R., Leveritt, M.D., and Aldred, M.J. (2008). Influence of closed skill and open skill warm-ups on the performance of speed, change of direction speed, vertical jump, and reactive agility in team sport athletes. J Strength Cond Res 22(5), 1413-1415. doi: 10.1519/JSC.0b013e3181739ecd.

Halouani, J., Chtourou, H., Gabbett, T., Chaouachi, A., and Chamari, K. (2014). Small-sided games in team sports training: a brief review. J Strength Cond Res 28(12), 3594-3618. doi: $10.1519 /$ jsc.0000000000000564.

Hedges, L.V. (1981). Distribution Theory for Glass's Estimator of Effect Size and Related Estimators. Journal of Educational Statistics 6(2), 107-128. doi: 10.2307/1164588. 
Iacono, A.D., Eliakim, A., and Meckel, Y. (2015). Improving fitness of elite handball players: small-sided games vs. high-intensity intermittent training. J Strength Cond Res 29(3), 835-843. doi: 10.1519/jsc.0000000000000686.

Johnston, R.D., Gibson, N.V., Twist, C., Gabbett, T.J., MacNay, S.A., and MacFarlane, N.G. (2013). Physiological responses to an intensified period of rugby league competition. $J$ Strength Cond Res 27(3), 643-654. doi: 10.1519/JSC.0b013e31825bb469.

Kawakami, Y., Muraoka, T., Ito, S., Kanehisa, H., and Fukunaga, T. (2002). In vivo muscle fibre behaviour during counter-movement exercise in humans reveals a significant role for tendon elasticity. $J$ Physiol 540(Pt 2), 635-646.

Lake, J., Lauder, M., Smith, N., and Shorter, K. (2012). A comparison of ballistic and nonballistic lower-body resistance exercise and the methods used to identify their positive lifting phases. J Appl Biomech 28(4), 431-437.

Lakens, D. (2013). Calculating and reporting effect sizes to facilitate cumulative science: a practical primer for t-tests and ANOVAs. Front Psychol 4, 863. doi: 10.3389/fpsyg.2013.00863.

Mahlfeld, K., Franke, J., and Awiszus, F. (2004). Postcontraction changes of muscle architecture in human quadriceps muscle. Muscle Nerve 29(4), 597-600. doi: 10.1002/mus.20021.

McGowan, C.J., Pyne, D.B., Thompson, K.G., and Rattray, B. (2015). Warm-Up Strategies for Sport and Exercise: Mechanisms and Applications. Sports Med 45(11), 1523-1546. doi: 10.1007/s40279-015-0376-x.

McShane, B.B., Gal, D., Gelman, A., Robert, C., Tackett, J.L. (2017). Abandon statistical significance. arXiv e-print.

Newton, R.U., Murphy, A.J., Humphries, B.J., Wilson, G.J., Kraemer, W.J., and Hakkinen, K. (1997). Influence of load and stretch shortening cycle on the kinematics, kinetics and muscle activation that occurs during explosive upper-body movements. Eur J Appl Physiol Occup Physiol 75(4), 333-342. doi: 10.1007/s004210050169.

Romaratezabala, E., Nakamura, F.Y., Castillo, D., Gorostegi-Anduaga, I., and Yanci, J. (2018). Influence of warm-up duration on physical performance and psychological perceptions in handball players. Res Sports Med, 1-14. doi: 10.1080/15438627.2018.1431536.

Schulz, K.F., and Grimes, D.A. (2002). Blinding in randomised trials: hiding who got what. Lancet 359(9307), 696-700. doi: 10.1016/s0140-6736(02)07816-9.

Souhail, H., Castagna, C., Mohamed, H.Y., Younes, H., and Chamari, K. (2010). Direct validity of the yo-yo intermittent recovery test in young team handball players. J Strength Cond Res 24(2), 465-470. doi: 10.1519/JSC.0b013e3181c06827.

Suchomel, T.J., Lamont, H.S., and Moir, G.L. (2016). Understanding Vertical Jump Potentiation: A Deterministic Model. Sports Med 46(6), 809-828. doi: 10.1007/s40279-015-0466-9.

Team, R.C. (2013). R: A language and environment for statistical computing.

Tillin, N.A., and Bishop, D. (2009). Factors modulating post-activation potentiation and its effect on performance of subsequent explosive activities. Sports Med 39(2), 147-166. doi: 10.2165/00007256-200939020-00004.

Wagner, H., and Muller, E. (2008). The effects of differential and variable training on the quality parameters of a handball throw. Sports Biomech 7(1), 54-71. doi: 10.1080/14763140701689822.

Wilson, J.M., Duncan, N.M., Marin, P.J., Brown, L.E., Loenneke, J.P., Wilson, S.M., et al. (2013). Meta-analysis of postactivation potentiation and power: effects of conditioning 
activity, volume, gender, rest periods, and training status. J Strength Cond Res 27(3), 854-859. doi: 10.1519/JSC.0b013e31825c2bdb.

Zois, J., Bishop, D.J., Ball, K., and Aughey, R.J. (2011). High-intensity warm-ups elicit superior performance to a current soccer warm-up routine. J Sci Med Sport 14(6), 522-528. doi: 10.1016/j.jsams.2011.03.012. 